\title{
Sexual Orientation Group Differences in Perceived Stress and Depressive Symptoms Among Young Adults in the United States
}

\author{
Evan A. Krueger, MPH, Ilan H. Meyer, PhD, and Dawn M. Upchurch, PhD, LAc ${ }^{1}$
}

\begin{abstract}
Purpose: Overall, sexual minorities have poorer mental health than heterosexual individuals, and stress is thought to underlie such disparities. However, sexual minorities include both those identifying as lesbian, gay, or bisexual (LGB) and many who do not (e.g., individuals identifying as mostly heterosexual, or as heterosexual but with discordant same-sex attractions or behaviors), and little is known about the mental health or stress experiences of non-LGB identified sexual minorities. This study assessed perceived stress and depressive symptom differences between concordant heterosexual individuals and three groups of sexual minority young adults (LGB, mostly heterosexual, and discordant heterosexual individuals).

Methods: Data were from the National Longitudinal Study of Adolescent to Adult Health, Wave IV (20082009). Descriptive and bivariate statistics were estimated. Path analyses assessed whether perceived stress mediated differences in depressive symptomatology. Analyses were weighted and gender-stratified.

Results: Mostly heterosexual individuals comprised the largest sexual minority group, for both men (3.58\%) and women $(15.88 \%)$. All sexual minority groups reported significantly more depressive symptoms than concordant heterosexual individuals, for both men and women (all $P<0.05$ ). Among women, all sexual minority groups reported significantly higher perceived stress than concordant heterosexual individuals (all $P<0.05$ ), which partially mediated elevations in depressive symptomatology (all $P<0.05$ ). Mostly-heterosexual-identified men reported significantly higher perceived stress than concordant heterosexual men $(P<0.01)$, which partially mediated elevations in depressive symptomatology $(P<0.01)$.

Conclusions: Our results underscore the importance of assessing sexual orientation comprehensively to fully understand sexual minority health disparities. Additional research should examine the stressors specific to different sexual minority groups.
\end{abstract}

Keywords: depressive symptoms, health disparities, perceived stress, sexual minority

\section{Introduction}

$\mathbf{S}$ EXUAL MiNORITIES (e.g., those who identify as lesbian, gay, or bisexual [LGB]) experience a wide range of health disparities compared to heterosexual individuals, ${ }^{1}$ with such disparities extending to young adults. ${ }^{2-4}$ Disparities in depressive symptomatology are also well documented, with sexual minorities reporting more symptoms and diagnoses than heterosexual individuals, especially among youths. ${ }^{5-7}$ LGB mental health inequalities are hypothesized to be driven largely by exposure to social stressors, including minorityspecific stressors stemming from prejudice and stigma, placing
LGB individuals at increased risk for depressive symptoms, compared to heterosexuals. $^{8-12}$

Studies of health disparities start with population definitions and measurement, allowing us to compare among groups. However, inconsistencies in the measurement of sexual identity have led to the exclusion of some sexual minorities who may be relevant to this discussion. Sexuality is determined through a developmental process marked by fluidity, especially during adolescence and young adulthood, ${ }^{13,14}$ yet in surveys, sexual identity is often defined using categories (e.g., LGB, heterosexual) that do not capture the complexity of sexual orientation. ${ }^{13,15}$ For example, a sizeable proportion

\footnotetext{
${ }^{1}$ Department of Community Health Sciences, Fielding School of Public Health, University of California, Los Angeles, Los Angeles, California.

${ }^{2}$ The Williams Institute, School of Law, University of California, Los Angeles, Los Angeles, California.
} 
of individuals describe themselves as "mostly heterosexual," rather than as completely heterosexual or as bisexual. ${ }^{16}$ However, while it is increasingly accepted that mostly heterosexual individuals form a distinct (and sizeable) sexual identity group, the degree to which they are different from exclusively heterosexual individuals is not well-understood, ${ }^{16,17}$ and due to measurement limitations, they are often excluded from relevant research. $^{16}$

Furthermore, sexual orientation is multidimensional, consisting of individuals' sexual identities, attractions, and behaviors. ${ }^{13,18-20}$ The term "sexual minority" refers to those with nonheterosexual identities, attractions, and/or behaviors, ${ }^{21}$ but single-indicator measures, such as identity, are often relied upon to distinguish sexual minorities from nonminorities. ${ }^{14,21}$ Moreover, sexual orientation dimensions often do not align. ${ }^{13,18,19}$ For example, "discordant heterosexual" persons are those who identify as heterosexual despite reporting same-sex attractions and/or engaging in samesex sexual behaviors. Based on their sexual identities alone, discordant heterosexual individuals may, thus, be incorrectly categorized together with heterosexuals with no same-sex attractions or behaviors. Use of single-indicator measures may lead to misclassification of people who are sexual minorities, and to biased estimates of sexual minority health disparities. $7,18,20,22,23$

Because much of the existing research on sexual minority mental health compares self-identified sexual minorities to self-identified heterosexuals, relatively little is known about the mental health statuses, or the stress experiences, of sexual minorities who do not identify as such (e.g., mostly heterosexual and discordant heterosexual individuals). While some research has shown that mostly heterosexual individuals carry increased risk for mental health and substance use disorders, ${ }^{17,24-26}$ very few representative studies have explicitly described the mental health of discordant heterosexual individuals. ${ }^{25,27}$ Furthermore, despite its theoretical relevance, ${ }^{8}$ to our knowledge, no studies have formally assessed the degree to which stress underlies any mental health hardships that may exist for either of these understudied populations.

The current study expands upon existing research in several ways. First, using nationally representative data, we explore whether differences in perceived stress exist between heterosexuals with concordant (i.e., opposite-sex only) attractions and behaviors and three sexual minority groups with variations in sexual identification: those identifying as LGB, as mostly heterosexual, or as heterosexual but with discordant behaviors or attractions. Minority Stress Theory suggests that sexual minority disparities in mental health status result from increased exposure to social stressors related to prejudice and stigma. ${ }^{8}$ Given their sexual minority status by at least one dimension, all sexual minority groups are hypothesized to experience increased stress exposure, compared with concordant heterosexual individuals. However, there may be differences between sexual minority groups. For example, LGB-identified sexual minorities may be more attuned to sexual orientation-related stress and therefore report more overall stress than mostly heterosexual and discordant heterosexual individuals, who may be shielded somewhat from identity-based victimization. Second, consistent with Minority Stress Theory, we hypothesize that increased levels of perceived stress will mediate depressive symptomatology disparities among sexual minorities, compared with concordant heterosexual individuals. ${ }^{8}$ Finally, given known gender differences in exposure to both chronic stressors and victimization, ${ }^{28}$ including sexual orientationrelated stigma, ${ }^{29}$ we examine men and women separately.

\section{Methods}

\section{Study design}

The National Longitudinal Study of Adolescent to Adult Health (Add Health) is a nationally representative, longitudinal sample of adolescents enrolled in grades 7-12 in 19941995. Using a multistage sampling design, 20,754 individuals were selected at Wave I to participate in in-home interviews, to be conducted longitudinally over time. Data for the present study were drawn from Wave IV of data collection, completed in 2008-2009, when respondents were 2434 years old $(N=15,701) .{ }^{30}$ Individuals were excluded from analysis if they were missing one or more of the following variables, described in detail below: sample weight $(N=901)$, depressive symptoms $(N=10)$, perceived stress $(N=28)$, sexual orientation group $(N=334)$, or age $(N=6)$. Those who identified as Native American $(N=115)$ or "other" $(N=143)$ races were excluded due to heterogeneity within the groups. The final analytic sample was 14,216 (6,637 men, 7,579 women). Add Health participants provided written informed consent, and the study was approved by the University of North Carolina at Chapel Hill Office of Human Research Ethics. ${ }^{30}$ The present study was approved by the University of California, Los Angeles Office of the Human Research Protection Program.

\section{Study variables}

Sexual identity was measured using the question, "Please choose the description that best fits how you think about yourself."' Responses were: "100\% heterosexual (straight)," "mostly heterosexual (straight), but somewhat attracted to your own sex," "bisexual, that is, attracted to men and women equally," "mostly homosexual (gay), but somewhat attracted to the opposite sex," "100\% homosexual (gay)," and "not sexually attracted to either males or females." Respondents were categorized as heterosexual (100\% heterosexual), mostly heterosexual, or LGB (bisexual, mostly homosexual, and $100 \%$ homosexual). Respondents missing sexual identity responses, and those reporting that they were "not sexually attracted to either males or females" were categorized as missing.

Sexual behavior was measured using the following questions: "Considering all types of sexual activity, with how many male partners have you ever had sex?" and "Considering all types of sexual activity, with how many female partners have you ever had sex?' Responses were compared to respondents' self-reported genders to assess same-sex directionality. Men who engaged in sexual activity with male partners, or with both male and female partners, and women who engaged in sexual activity with female partners, or with both male and female partners, were coded as having engaged in same-sex behavior. Respondents with no same-sex partners were coded as such. Men and women missing responses for numbers of male and female sex partners, respectively, were categorized as missing. 
Sexual attraction was measured using the following questions: "Are you romantically attracted to males?" and "Are you romantically attracted to females?" Participants responded "yes" or "no." Men reporting attraction to males, women reporting attraction to females, and all respondents reporting attraction to both males and females were coded as having same-sex attraction. Respondents reporting attraction to only different-sex partners were coded as such. Men and women missing responses for attraction to males and females, respectively, and respondents not attracted to either males or females were categorized as missing.

Sexual orientation groups. Based on their sexual identities, behaviors, and attractions, respondents were categorized into one of four sexual orientation groups. Heterosexuals with concordant (opposite-sex) attractions and concordant (no prior same-sex) sexual behavior served as the comparison group. The remaining respondents were categorized into one of three sexual minority groups: LGB-identified individuals, mostly heterosexual-identified individuals, and heterosexual-identified individuals who also endorsed discordant (same-sex) attractions and/or behaviors. Three hundred and thirty-four respondents were missing the identity, behavior, and/or attraction variables necessary for categorization into sexual orientation groups and were excluded from analysis.

Depressive symptoms were measured using five items from the Center for Epidemiologic Studies Depression Scale measure of depressive symptomatology, a frequently-used and validated measure of the construct. ${ }^{31,32}$ The frequency of each of the following items was assessed in the past 7 days: "You were bothered by things that don't usually bother you," "You could not shake off the blues, even with help from your family and your friends," "You felt you were just as good as other people," "You had trouble keeping your mind on what you were doing," and "You felt depressed." Participants responded on a Likert scale ("never or rarely," "sometimes," "'a lot of the time," or "most of the time or all of the time"). Items were reversecoded as necessary. Final scores ranged from 0 (no depressive symptoms) to 15 (maximum).

Perceived stress was assessed using the four item version of the Cohen Perceived Stress Scale, a reasonably reliable and well-validated measure (Cronbach's alpha=0.67). ${ }^{33,34}$ The items were, "In the last 30 days, how often have you felt that you were unable to control the important things in your life?," "In the last 30 days, how often have you felt confident in your ability to handle your personal problems?," "In the last 30 days, how often have you felt that things were going your way?," and "In the last 30 days, how often have you felt that difficulties were piling up so high that you could not overcome them?" Participants reported how frequently ("never," "almost never," "sometimes," "fairly often," or "very often") they experienced each of the stressors. Items were reverse-coded as necessary. Total scores ranged from 0 (low stress) to 16 (high stress).

Demographic characteristics. Gender was measured dichotomously (male, female). Age was measured as a continuous count in years. Race/ethnicity was based on self-report (non-Hispanic white, non-Hispanic black, non-Hispanic Asian, Hispanic). Those who endorsed a Hispanic ethnicity were categorized as Hispanic, regardless of race. Nativity status was a dichotomous variable (born a US citizen vs. not). Educational attainment (less than high school, high school/General Equivalency Diploma, some college, 4-year college, more than college) and annual household income $(<\$ 25,000, \$ 25,000-\$ 49,999, \$ 50,000-\$ 74,999, \geq \$ 75,000)$ were coded as ordinal variables. Missing income values were imputed using regression imputation.

\section{Data analysis}

Bivariate differences in depressive symptoms and perceived stress, and demographic differences were assessed between sexual orientation groups using Stata $14 .^{35}$ Wald tests assessed differences for continuous variables, and design-based $F$ tests assessed differences for categorical variables. Next, using MPlus $7,{ }^{36}$ path analyses ${ }^{37}$ assessed differences in perceived stress and depressive symptomatology between concordant heterosexuals and each sexual minority group. Total, direct, and indirect effects were also estimated. Total effects models assessed depressive symptomatology differences between sexual orientation groups. Direct effects models assessed these associations, independent of potential mediating effects through perceived stress. Indirect effects models assessed the degree to which the associations were explained by (i.e., mediated through) perceived stress. All path analyses controlled for age, race/ ethnicity, nativity, education, and income. All analyses used survey weights to account for the complex survey design, and to allow for generalization to the U.S. population of young adults. Finally, all analyses were performed separately by gender.

\section{Results}

Table 1 displays mean depressive symptom and perceived stress scores, and demographic characteristics, separately by sexual orientation group among men. Concordant heterosexual men were the largest group $(90.57 \%)$, followed by mostly heterosexual men (3.58\%). Gay/bisexual and discordant heterosexual men each comprised $2.93 \%$ of the sample. Concordant heterosexual men had the fewest mean number of depressive symptoms (2.29) followed by gay/bisexual (2.64), mostly heterosexual (3.03), and discordant heterosexual men (3.22) $(P<0.001)$. Concordant heterosexual men reported the lowest mean perceived stress (4.52) followed closely by gay/bisexual men (4.74), while discordant heterosexual and mostly heterosexual men reported the same mean score (5.29) $(P=0.005)$. No age differences were present. Higher percentages of discordant heterosexual men were Hispanic and black and fewer were white, compared to other sexual orientation groups. Gay/bisexual and mostly heterosexual men reported higher educational attainment than other groups. However, a higher percentage of concordant heterosexual men had higher incomes, and a higher percentage of discordant heterosexual men had lower incomes, compared to other groups.

Table 2 displays mean depressive symptom and perceived stress scores, and demographic characteristics, separately by sexual orientation group among women. Among women, concordant heterosexual individuals were the largest group (75.31\%), followed by mostly heterosexual $(15.88 \%)$, discordant heterosexual (4.62\%), and LGB individuals (4.19\%). 
Table 1. Selected Characteristics by Sexual Orientation Group, Men, Add Health Wave IV, 2008-2009 $(N=6,637)$

\begin{tabular}{|c|c|c|c|c|c|}
\hline & $\begin{array}{l}\text { Concordant } \\
\text { heterosexual }\end{array}$ & Gay, bisexual & $\begin{array}{c}\text { Mostly } \\
\text { heterosexual }\end{array}$ & $\begin{array}{l}\text { Discordant } \\
\text { heterosexual }\end{array}$ & \\
\hline (N) weighted\% & $(\mathrm{N}=6,000) 90.57 \%$ & $(\mathrm{~N}=223) 2.93 \%$ & $(\mathrm{~N}=228) 3.58 \%$ & $(\mathrm{~N}=186) 2.93 \%$ & $\mathrm{P}$ \\
\hline $\begin{array}{l}\text { Depressive symptoms } \\
\text { (mean, range: } 0-15)\end{array}$ & 2.29 & 2.64 & 3.03 & 3.22 & $<0.001$ \\
\hline Perceived stress (mean, range: $0-16$ ) & 4.52 & 4.74 & 5.29 & 5.29 & 0.005 \\
\hline Age (mean) & 28.91 & 28.91 & 28.91 & 29.26 & 0.307 \\
\hline Race/ethnicity (\%) & & & & & 0.004 \\
\hline Hispanic & 11.45 & 17.75 & 12.09 & 20.82 & \\
\hline Non-Hispanic black & 15.38 & 13.07 & 8.77 & 23.92 & \\
\hline Non-Hispanic Asian & 3.46 & 3.99 & 3.41 & 0.98 & \\
\hline Non-Hispanic white & 69.71 & 65.19 & 75.74 & 54.28 & \\
\hline Nativity (born a US citizen) (\%) & 96.03 & 94.02 & 98.33 & 91.11 & 0.021 \\
\hline Education $(\%)$ & & & & & $<0.001$ \\
\hline$<$ High school & 10.34 & 7.99 & 6.96 & 15.06 & \\
\hline High school/GED & 30.32 & 18.94 & 18.62 & 44.35 & \\
\hline Some college & 32.45 & 34.44 & 31.84 & 29.62 & \\
\hline 4-year college & 18.04 & 23.49 & 31.49 & 5.16 & \\
\hline More than college & 8.84 & 15.14 & 11.09 & 5.81 & \\
\hline Household income (\%) & & & & & $<0.001$ \\
\hline$<\$ 25,000$ & 14.46 & 14.87 & 24.54 & 29.49 & \\
\hline$\$ 25,000-\$ 49,999$ & 31.14 & 32.70 & 35.08 & 34.64 & \\
\hline$\$ 50,000-\$ 74,999$ & 23.82 & 22.94 & 15.10 & 11.25 & \\
\hline$\geq \$ 75,000$ & 30.58 & 29.49 & 25.27 & 24.62 & \\
\hline
\end{tabular}

The table presents weighted means and percentages. Adjusted Wald tests were performed to calculate $P$ values for continuous variables, and design-based $F$ tests were performed to calculate $P$ values for categorical variables.

GED, general equivalency diploma.

Table 2. Selected Characteristics by Sexual Orientation Group, Women, Add Health Wave IV, 2008-2009 $(N=7,579)$

\begin{tabular}{|c|c|c|c|c|c|}
\hline & $\begin{array}{l}\text { Concordant } \\
\text { Heterosexual }\end{array}$ & $\begin{array}{l}\text { Lesbian, Gay, } \\
\text { Bisexual }\end{array}$ & $\begin{array}{c}\text { Mostly } \\
\text { Heterosexual }\end{array}$ & $\begin{array}{l}\text { Discordant } \\
\text { Heterosexual }\end{array}$ & \\
\hline (N) weighted\% & $(\mathrm{N}=5,756) 75.31 \%$ & $(\mathrm{~N}=316) 4.19 \%$ & $(\mathrm{~N}=1,178) 15.88 \%$ & $(\mathrm{~N}=329) 4.62 \%$ & $\mathrm{P}$ \\
\hline $\begin{array}{l}\text { Depressive symptoms } \\
\text { (mean, range: } 0-15)\end{array}$ & 2.62 & 4.09 & 3.43 & 3.63 & $<0.001$ \\
\hline Perceived stress (mean, range: $0-16$ ) & 4.80 & 6.26 & 5.91 & 5.53 & $<0.001$ \\
\hline Age (mean) & 28.80 & 28.38 & 28.44 & 28.63 & $<0.001$ \\
\hline Race/ethnicity (\%) & & & & & $<0.001$ \\
\hline Hispanic & 11.72 & 13.79 & 10.62 & 14.24 & \\
\hline Non-Hispanic black & 17.65 & 17.03 & 9.20 & 14.14 & \\
\hline Non-Hispanic Asian & 3.17 & 1.48 & 2.81 & 4.66 & \\
\hline Non-Hispanic white & 67.46 & 67.71 & 77.37 & 66.95 & \\
\hline Nativity (born a US citizen) (\%) & 95.67 & 96.51 & 97.98 & 94.80 & 0.023 \\
\hline Education $(\%)$ & & & & & $<0.001$ \\
\hline$<$ High school & 6.49 & 15.48 & 6.83 & 11.24 & \\
\hline High school/GED & 22.88 & 28.13 & 22.78 & 29.35 & \\
\hline Some college & 34.62 & 36.63 & 38.69 & 33.30 & \\
\hline 4-year college & 21.08 & 13.85 & 18.14 & 19.61 & \\
\hline More than college & 14.94 & 5.91 & 13.57 & 6.51 & \\
\hline Household income (\%) & & & & & $<0.001$ \\
\hline$<\$ 25,000$ & 18.40 & 36.02 & 17.81 & 24.00 & \\
\hline$\$ 25,000-\$ 49,999$ & 30.56 & 31.88 & 31.04 & 34.04 & \\
\hline$\$ 50,000-\$ 74,999$ & 23.21 & 17.28 & 25.47 & 19.28 & \\
\hline$\geq \$ 75,000$ & 27.83 & 14.82 & 25.68 & 22.68 & \\
\hline
\end{tabular}

The table presents weighted means and percentages. Adjusted Wald tests were performed to calculate $P$ values for continuous variables, and design-based $F$ tests were performed to calculate $P$ values for categorical variables. 

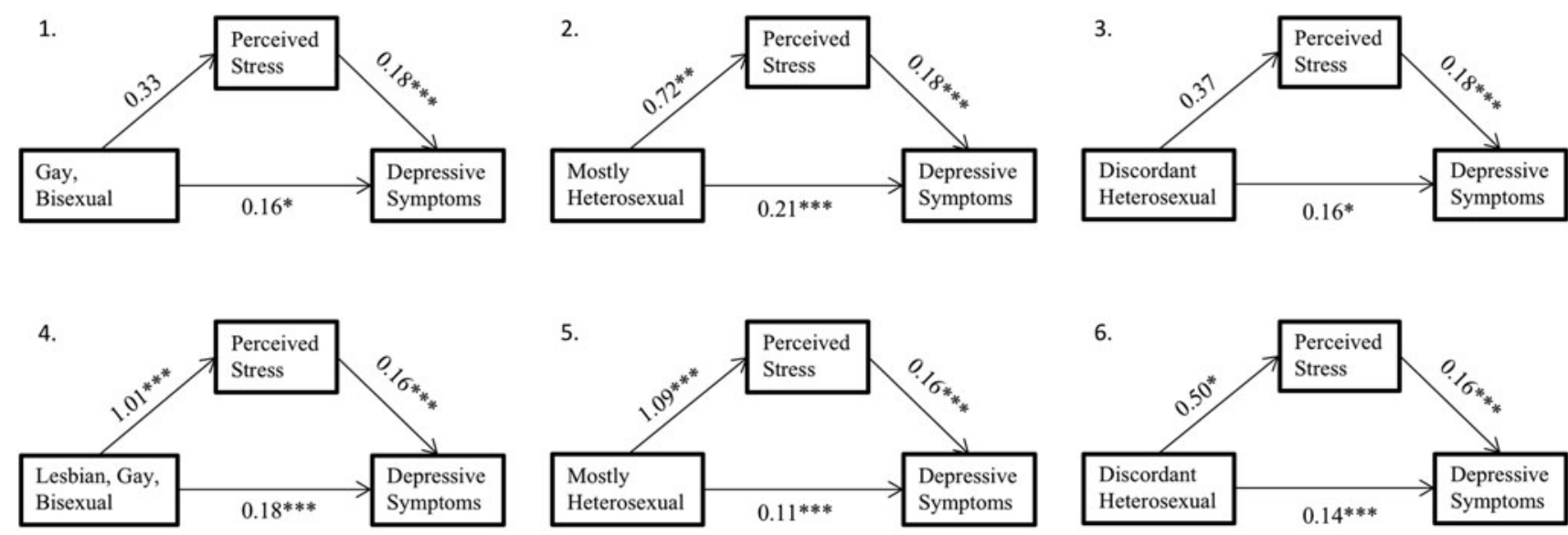

FIG. 1. Associations between sexual orientation group, perceived stress, and depressive symptoms: Path coefficients, weighted path analyses, Add Health Wave IV, 2008-2009 ( $N=6,637$ men, 7,579 women). Values reported are path coefficients. All analyses controlled for sex, age, race/ethnicity, nativity, educational attainment, and household income. $* * * P<0.001 ; * * P<0.01 ; * P<0.05$. 1. Gay/bisexual, compared with concordant heterosexual, men; 2 . Mostly heterosexual, compared with concordant heterosexual, men; 3. Discordant heterosexual, compared with concordant heterosexual, men; 4. Lesbian/gay/bisexual, compared with concordant heterosexual, women; 5. Mostly heterosexual, compared with concordant heterosexual, women; and 6. Discordant heterosexual, compared with concordant heterosexual, women.

Concordant heterosexual women had the fewest mean number of depressive symptoms (2.62) followed by mostly heterosexual (3.43), discordant heterosexual (3.63), and LGB women $(4.09) \quad(P<0.001)$. Concordant heterosexual women reported the lowest mean perceived stress (4.80) followed by discordant heterosexual (5.53), mostly heterosexual (5.91), and LGB women (6.26) $(P<0.001)$. Small, but significant age differences existed between groups. Higher percentages of mostly heterosexual women were white and were born as U.S. citizens, compared with other sexual orientation groups. Concordant heterosexual and mostly heterosexual women reported higher educational attainment than other groups. Finally, a higher percentage of concordant heterosexual women had higher incomes, and a higher percentage of LGB women had lower incomes, compared with other groups.

Figure 1 presents individual path coefficients from a series of path analyses, controlling for covariates. Among both men and women, each sexual minority group had more depressive symptoms than concordant heterosexuals (all $P<0.05$ ). For both men and women, increased perceived stress was associated with more depressive symptoms (all $P<0.001$ ). Among women, each sexual minority group reported higher perceived stress than concordant heterosexual individuals (all $P<0.05)$. However, only mostly heterosexual men reported higher perceived stress than concordant heterosexual men $(P<0.01)$.

Table 3 presents the total, direct, and indirect effect estimates from the path analyses. Among women, all sexual minority subgroups had significant total, direct, and indirect effects (i.e., sexual minority groups had increased perceived stress, which in turn, was associated with more depressive symptoms), compared to concordant heterosexual individuals (all $P<0.05$ ). However, among men, while all sexual minority groups had significant total and direct effects (all $P<0.05$ ), only mostly heterosexual individuals had significant indirect effects $(P<0.01)$, compared with concordant heterosexual individuals.

\section{Discussion}

Our results highlight several variations that exist in sexual identification among sexual minorities and underscore the importance of comprehensively assessing multiple sexual orientation dimensions to fully understand sexual orientation health differences. Among both men and women, all sexual minority groups-LGB, mostly heterosexual, and discordant heterosexual respondents-experienced more depressive symptoms than concordant heterosexual respondents. While consistent with research showing mental health disparities between heterosexual and LGB people, ${ }^{5-7}$ our findings also show that frequently excluded sexual minority groups-mostly heterosexual and discordant heterosexual

Table 3. Mediating Effect of Perceived Stress on the Associations Between Sexual Orientation Groups ANd Depressive Symptomatology, Weighted Path Analyses, Add Health Wave IV, 2008-2009. $(N=6,637$ MEN, 7,579 WoMEN $)$

\begin{tabular}{lll}
\hline & Men, $B(S E)$ & Women, $B(S E)$ \\
\hline $\begin{array}{l}\text { Lesbian, gay, bisexual } \\
\text { Total effect }\end{array}$ & $0.22(0.09)^{* * *}$ & $0.34(0.06)^{* * *}$ \\
Direct effect & $0.16(0.07)^{*}$ & $0.18(0.05)^{* * *}$ \\
Indirect effect & $0.06(0.04)$ & $0.17(0.05)^{* * *}$ \\
Mostly heterosexual & & \\
Total effect & $0.34(0.07)^{* * *}$ & $0.29(0.03)^{* * *}$ \\
Direct effect & $0.21(0.06)^{* * *}$ & $0.11(0.03)^{* * *}$ \\
Indirect effect & $0.13(0.05)^{* *}$ & $0.18(0.02)^{* * *}$ \\
Discordant heterosexual & \\
Total effect & $0.23(0.07)^{* * *}$ & $0.22(0.06)^{* * *}$ \\
Direct effect & $0.16(0.07)^{*}$ & $0.14(0.04)^{* * *}$ \\
Indirect effect & $0.07(0.05)$ & $0.08(0.04)^{*}$ \\
\hline
\end{tabular}

For all models, concordant heterosexuals are the referent group. All models controlled for sex, age, race/ethnicity, nativity status, educational attainment, and household income.

$* * * P<0.001 ; * * P<0.01 ; * P<0.05$. 
individuals-experience similar mental health disparities to LGB-identified individuals. Furthermore, several sociodemographic differences (e.g., race/ethnicity, education) were present across sexual minority groups, highlighting important intersections between sexual identity and other identities and characteristics, which should be explored further.

These results further show that, among young adults, the mostly heterosexual and discordant heterosexual populations are as large, or larger, than the population of LGB-identified individuals, and that, at least in terms of perceived stress and depressive symptoms, they are more similar to LGB individuals than concordant heterosexual individuals. This suggests that measures relying on identity alone may misclassify young sexual minority individuals who identify as heterosexual, masking the extent of sexual minority mental health disparities, and impeding researchers' and practitioners' abilities to assess and intervene with the full sexual minority population.

As the purpose of this study was to compare sexual minorities identifying as such to those who do not, LGB-identified sexual minorities were combined into a single analytic group. However, it will be important for future research to examine differences between sexual minority identities. For instance, there is growing evidence that bisexuals experience differential and often deeper health disparities than gay and lesbian individuals. ${ }^{38,39}$

\section{Gender differences}

Although women and men were not compared directly, results from our stratified analyses suggest that gender differences exist in stress and mental health processes among sexual minorities. Compared to men, women were approximately 4.5 times as likely to identify as mostly heterosexual, and were approximately 1.6 times as likely to be discordantly heterosexual, consistent with previous research showing that young women express more variability and fluidity than men in the use of sexual identity labels. ${ }^{20}$ Furthermore, among women, all sexual minority groups reported higher perceived stress than concordant heterosexual individuals, which partially mediated increases in depressive symptomatology. These findings are consistent with Minority Stress Theory, which asserts that increased social stress underlies mental health disparities among sexual minorities. ${ }^{8}$ However, among men, only the results for mostly heterosexual men fully supported minority stress hypotheses. Although gay/bisexual-identified men and discordant heterosexual men reported more depressive symptoms than concordant heterosexual men, they did not report more perceived stress, nor did stress mediate the relationship between sexual orientation and depressive symptomatology.

The gender differences that we reported in the impact of perceived stress on depressive symptoms might represent a limitation of our measure of stress, which assessed general stress rather than specific minority stress processes. Although perceived stress mediated mental health disparities for all groups of sexual minority women measured here and among mostly heterosexual men, it may have a more tenuous role among gay/bisexual and discordant heterosexual men. It is possible that alternative or more refined measures of stress (e.g., minority-specific stressors, including prejudice events, internalized homophobia, or expectations of rejection ${ }^{8,40}$ ) would show the hypothesized full mediation of depressive symptom disparities for these groups. Additional research is needed to assess these hypotheses.

Furthermore, while all sexual minorities may experience stress associated with decisions to reveal or conceal aspects of their sexual orientations (identity, attractions, and/or behaviors), ${ }^{24}$ it is also possible that group differences exist in exposure to other stressors. For example, salient stressors for LGB-identified individuals might include harassment, whereas more salient stressors for discordant and mostly heterosexual individuals might include isolation from similar others. To address health disparities meaningfully, future studies should assess, more comprehensively, differences in the types of stressors most salient to different sexual minority groups.

\section{Limitations}

There are analytic limitations of our study. Although this article advocates for assessing sexual orientation comprehensively, we were limited by the items available in Add Health. For example, the response options available for the identity term confound identity with attraction [e.g., "mostly heterosexual (straight), but somewhat attracted to your own sex"]; and the measure included the term "gay" as an example of "homosexual," but not other nonheterosexual identities (e.g., lesbian, queer). In addition, sample size concerns led to excluding Native American and "other" races, because no meaningful analyses could be performed on these groups. These data were collected in 2008-2009, and so do not account for recent social changes surrounding the acceptance of sexual diversity, or possible changes in the use of identity labels. Finally, these data are cross-sectional, limiting our ability to draw inferences about the causal nature of the associations we presented.

\section{Conclusion}

This study highlighted several important intersections between sexual identity, attraction, behavior, and gender. To our knowledge, this is the first study to formally assess stress as a mechanism by which two understudied sexual minority groups-mostly heterosexual and discordant heterosexual individuals-experience disparities in depressive symptomatology, particularly among women. It shows that in addition to LGB-identified persons, discordant heterosexual and mostly heterosexual individuals should be studied as at risk, sexual minority populations.

\section{Acknowledgments}

This research uses data from Add Health, a program project directed by Kathleen Mullan Harris and designed by J. Richard Udry, Peter S. Bearman, and Kathleen Mullan Harris at the University of North Carolina at Chapel Hill and funded by grant P01-HD31921 from the Eunice Kennedy Shriver National Institute of Child Health and Human Development, with cooperative funding from 23 other federal agencies and foundations. Special acknowledgment is due to Ronald R. Rindfuss and Barbara Entwisle for assistance in the original design. Information on how to obtain the Add Health data files is available on the Add Health website (www.cpc .unc.edu/addhealth). No direct support was received from grant P01-HD31921 for this analysis. This project was 
supported by a predoctoral fellowship from the UCLA Graduate Division, and awards from the National Institute on Aging (T32 AG033533), and the Eunice Kennedy Shriver National Institute of Child Health and Human Development (R24 HD041022, R01 HD078526).

\section{Disclaimer}

The content of this article is solely the responsibility of the authors and does not necessarily represent the official views of the funding agencies.

\section{Author Disclosure Statement}

No competing financial interests exist.

\section{References}

1. Institute of Medicine (US) Committee on Lesbian, Gay, Bisexual, and Transgender Health Issues and Research Gaps and Opportunities: The Health of Lesbian, Gay, Bisexual, and Transgender People: Building a Foundation for Better Understanding. Washington, DC: National Academies Press (US), 2011.

2. McLaughlin KA, Hatzenbuehler ML, Xuan Z, Conron KJ: Disproportionate exposure to early-life adversity and sexual orientation disparities in psychiatric morbidity. Child Abuse Negl 2012;36:645-655.

3. Russell ST, Joyner K: Adolescent sexual orientation and suicide risk: Evidence from a national study. Am J Public Health 2001;91:1276-1281.

4. Russell ST, Ryan C, Toomey RB, et al.: Lesbian, gay, bisexual, and transgender adolescent school victimization: Implications for young adult health and adjustment. J Sch Health 2011;81:223-230.

5. Almeida J, Johnson RM, Corliss HL, et al.: Emotional distress among LGBT youth: The influence of perceived discrimination based on sexual orientation. J Youth Adolesc 2009;38:1001-1014.

6. Marshal MP, Dietz LJ, Friedman MS, et al.: Suicidality and depression disparities between sexual minority and heterosexual youth: A meta-analytic review. J Adolesc Health 2011;49:115-123.

7. Lindley LL, Walsemann KM, Carter JW Jr: The association of sexual orientation measures with young adults' health-related outcomes. Am J Public Health 2012;102: 1177-1185.

8. Meyer IH: Prejudice, social stress, and mental health in lesbian, gay, and bisexual populations: Conceptual issues and research evidence. Psychol Bull 2003;129:674-697.

9. Meyer IH: Minority stress and mental health in gay men. J Health Soc Behav 1995;36:38-56.

10. Hatzenbuehler ML: The social environment and suicide attempts in lesbian, gay, and bisexual youth. Pediatrics 2011; 127:896-903.

11. Hatzenbuehler ML: Social factors as determinants of mental health disparities in LGB populations: Implications for public policy. Soc Issues Policy Rev 2010;4:31-62.

12. Diamond GM, Shilo G, Jurgensen E, et al.: How depressed and suicidal sexual minority adolescents understand the causes of their distress. J Gay Lesbian Ment Health 2011; 15:130-151.

13. Igartua K, Thombs BD, Burgos G, Montoro R: Concordance and discrepancy in sexual identity, attraction, and behavior among adolescents. J Adolesc Health 2009;45:602-608.
14. Rosario M, Schrimshaw EW, Hunter J, Braun L: Sexual identity development among gay, lesbian, and bisexual youths: Consistency and change over time. J Sex Res 2006;43:46-58.

15. Vrangalova Z, Savin-Williams RC: Mostly heterosexual and mostly gay/lesbian: Evidence for new sexual orientation identities. Arch Sex Behav 2012;41:85-101.

16. Savin-Williams RC, Vrangalova Z: Mostly heterosexual as a distinct sexual orientation group: A systematic review of the empirical evidence. Dev Rev 2013;33:58-88.

17. Kuyper L, Bos H: Mostly heterosexual and lesbian/gay young adults: Differences in mental health and substance use and the role of minority stress. J Sex Res 2016;53:731-741.

18. Korchmaros JD, Powell C, Stevens S: Chasing sexual orientation: A comparison of commonly used single-indicator measures of sexual orientation. J Homosex 2013;60:596614

19. Reback CJ, Larkins S: Maintaining a heterosexual identity: Sexual meanings among a sample of heterosexually identified men who have sex with men. Arch Sex Behav 2010;39: 766-773.

20. Johns MM, Zimmerman M, Bauermeister JA: Sexual attraction, sexual identity, and psychosocial wellbeing in a national sample of young women during emerging adulthood. J Youth Adolesc 2013;42:82-95.

21. Saewyc EM, Bauer GR, Skay CL, et al.: Measuring sexual orientation in adolescent health surveys: Evaluation of eight school-based surveys. J Adolesc Health 2004;35:345.e1-e15.

22. Young RM, Meyer IH: The trouble with "MSM" and "WSW": Erasure of the sexual-minority person in public health discourse. Am J Public Health 2005;95:1144-1149.

23. Muñoz-Laboy MA: Beyond "MSM': Sexual desire among bisexually-active Latino men in New York City. Sexualities 2004; 7:55-80.

24. Hughes TL, Wilsnack SC, Kristjanson AF: Substance use and related problems among U.S. women who identify as mostly heterosexual. BMC Public Health 2015;15:803.

25. Lourie MA, Needham BL: Sexual orientation discordance and young adult mental health. J Youth Adolesc 2017;46: 943-954.

26. Persson TJ, Pfaus JG, Ryder AG: Explaining mental health disparities for non-monosexual women: Abuse history and risky sex, or the burdens of non-disclosure? Soc Sci Med 2015; 128:366-373.

27. Cochran SD, Mays VM: Burden of psychiatric morbidity among lesbian, gay, and bisexual individuals in the California Quality of Life Survey. J Abnorm Psychol 2009; 118:647-658.

28. Nolen-Hoeksema S: Gender differences in depression. Curr Dir Psychol Sci 2001;10:173-176.

29. Herek GM: Sexual prejudice and gender: Do heterosexuals' attitudes toward lesbians and gay men differ? J Soc Issues 2000;56:251-266.

30. Harris KM: The Add Health Study: Design and Accomplishments. Carolina Population Center University of North Carolina at Chapel Hill, 2013. Available at www.cpc.unc.edu/ projects/addhealth/documentation/guides/DesignPaperWIIV .pdf Accessed July 2, 2015.

31. Radloff LS: The CES-D scale: A self-report depression scale for research in the general population. Appl Psychol Measure 1977;1:385-401.

32. Perreira KM, Deeb-Sossa N, Harris KM, Bollen K: What are we measuring? An evaluation of the CES-D across race/ethnicity and immigrant generation. Soc Forces 2005; 83:1567-1601. 
33. Cohen S, Kamarck T, Mermelstein R: A global measure of perceived stress. J Health Soc Behav 1983;24:385-396.

34. Cohen S, Williamson GM: Perceived stress in a probability sample of the United States. In: The Social Psychology of Health: The Claremont Symposium on Applied Social Psychology. Edited by Spacapan S, Oskamp S. Newbury Park, CA: Sage Publications, Inc., 1988, pp 31-67.

35. StataCorp: Stata Statistical Software: Release 14. College Station, TX: StataCorp LP, 2015.

36. Muthén LK, Muthén BO: MPlus User's Guide, 7th ed. Los Angeles, CA: Muthén \& Muthén, 1998-2012.

37. Lei PW, Wu Q: Introduction to structural equation modeling: Issues and practical considerations. Educ Meas Issues Pract 2007;26:33-43.

38. Balsam KF, Beauchaine TP, Mickey RM, Rothblum ED: Mental health of lesbian, gay, bisexual, and heterosexual siblings: Effects of gender, sexual orientation, and family. J Abnorm Psychol 2005;114:471-476.
39. Koh AS, Ross LK: Mental health issues: A comparison of lesbian, bisexual and heterosexual women. J Homosex 2006;51:33-57.

40. Meyer IH, Schwartz S, Frost DM: Social patterning of stress and coping: Does disadvantaged social statuses confer more stress and fewer coping resources? Soc Sci Med 2008;67: $368-379$.

Address correspondence to: Evan A. Krueger, $\mathrm{MPH}$ Department of Community Health Sciences Fielding School of Public Health University of California, Los Angeles 650 Charles E. Young Drive South Los Angeles, CA 90095

E-mail: eakrueger@ucla.edu 